\title{
Novel Mechanisms in Heart Failure With Preserved, Midrange, and Reduced Ejection Fraction
}

\author{
Ishan Lakhani ${ }^{1,2}$, Keith Sai Kit Leung ${ }^{1,2,3}$, Gary Tse ${ }^{1,2}$ and Alex Pui Wai Lee ${ }^{1 *}$ \\ ${ }^{1}$ Department of Medicine and Therapeutics, Faculty of Medicine, Chinese University of Hong Kong, Hong Kong, China, \\ ${ }^{2}$ Faculty of Medicine, Li Ka Shing Institute of Health Sciences, Chinese University of Hong Kong, Hong Kong, China, ${ }^{3}$ Aston \\ Medical School, Aston University, Birmingham, United Kingdom
}

Keywords: inflammation, phenomapping, LV dyssynchrony, network analysis, mortality

\section{INTRODUCTION}

OPEN ACCESS

Edited by:

Rui Plácido,

University of Lisbon, Portugal

Reviewed by:

Jens Spiesshoefer,

Universität Münster, Germany

Sang-Bing Ong,

Duke-NUS Medical School, Singapore Janos Paloczi

National Institutes of Health (NIH), United States

*Correspondence: Alex Pui Wai Lee alexpwlee@cuhk.edu.hk

Specialty section:

This article was submitted to Clinical and Translational Physiology, a section of the journal Frontiers in Physiology

Received: 21 February 2019 Accepted: 21 June 2019 Published: 05 July 2019

Citation:

Lakhani I, Leung KSK, Tse G and Lee APW (2019) Novel Mechanisms in Heart Failure With Preserved,

Midrange, and Reduced Ejection Fraction. Front. Physiol. 10:874. doi: 10.3389/fphys.2019.00874
Heart failure (HF) represents a major epidemic with high morbidity and mortality rates, imposing a significant burden on healthcare systems worldwide (Savarese and Lund, 2017). HF has long been distinguished by ejection fraction (EF) into two types-HF with reduced ejection fraction (HFrEF), for which $\mathrm{EF}$ is below $40 \%$, and $\mathrm{HF}$ with preserved ejection fraction (HFpEF), for which $\mathrm{EF}$ is above $50 \%$ and, according to the 2016 European Society of Cardiology (ESC) Guidelines (Ponikowski et al., 2016), accompanies (1) an elevated level of natriuretic peptides (BNP > 35 $\mathrm{pg} / \mathrm{ml}$ and/or NT-proBNP $>125 \mathrm{pg} / \mathrm{mL}$ ) and (2) the presence of either structural heart disease (left ventricular hypertrophy and/or left atrial enlargement) or diastolic dysfunction. HFrEF and HFpEF were initially considered to be binary opposing entities at two ends of the same spectrum. However, whilst several studies have demonstrated the efficacy of drug therapies in improving quality-of-life and long-term clinical outcomes in HFrEF patients, such pharmacological approaches have often failed to yield similar observable benefits in HFpEF cohorts. As such, the current paradigm follows that the pathogenesis underscoring the development and progression of HFrEF and HFpEF are distinct. In more recent developments, the 2016 ESC Guidelines (Ponikowski et al., 2016) also proposed a third class of HF-HF with mid-range ejection fraction (HFmrEF), for which EF is between 40 and 49\%, and accompanies the same two aforementioned components of HFpEF. Investigations into this newly defined group of HF patients have yielded contradicting results: whilst some findings have demonstrated an overlap between HFmrEF and the other two classes, others have shown no such association. As a result, a greater understanding of the underlying mechanistic differences between the HF groups, particularly pertaining to HFpEF and HFmrEF, is still needed in order to ensure successful diagnoses and holistic treatment provision.

The proposed mechanism for HFrEF is generally well-understood, in which adverse myocardial remodeling, resulting from cardiomyocyte death (Gonzalez et al., 2011) secondary to an inciting stimulus, such as viral myocarditis, myocardial infarction, or drug-induced cardiomyopathy (Bloom et al., 2017), leads to systolic dysfunction (Figure 1A). The same however cannot be said for HFpEF, which is instead associated with a more heterogeneous pathophysiology (Kao et al., 2015). Epidemiological studies have illustrated a comparatively stronger relationship between HFpEF (as opposed to HFrEF) with multiple cardiac and non-cardiac co-morbidities, including but not limited to type 2 diabetes mellitus (T2DM), arterial hypertension, renal failure, obesity, and atrial fibrillation (Elguindy and Yacoub, 2012). This evidently diverse clinical phenotype has elicited much debate regarding the precise mechanisms involved in the development of HFpEF. 


\section{SYSTEMIC PROINFLAMMATORY HYPOTHESIS}

One potential hypothesis suggests that HFpEF is simply the additive outcome of the many associated co-morbidities acting synergistically (Kao et al., 2015). Paulus et al. proposes a mechanism that lends credence to this notion by indicating that the concurrent existence of conditions such as T2DM, obesity, arterial hypertension, and pulmonary disease is responsible for inducing a systemic proinflammatory state (Figure 1B), characterized by elevated levels of tumor necrosis factor (TNF)$\alpha$, interleukin (IL)-6, and IL-1ß, amongst many others (Van Linthout and Tschöpe, 2017). Such cytokines in turn initiate a series of signaling events that ultimately culminate in reduced endothelial nitric oxide (NO) production and diminished activity of the cyclic guanosine phosphate-protein kinase G (cGMP$\mathrm{PKG}$ ) pathway in cardiomyocytes. This cascade of reactions eventually results in cardiomyocyte stiffness coupled with myocardial collagen deposition and fibrosis, therein leading to the development of hypertrophy, diastolic dysfunction and HFpEF (Paulus and Tschöpe, 2013). This theory has been supported not only by various animal models demonstrating the protective role of NO-cGMP-PKG signaling against myocardial hypertrophy (Calderone et al., 1998) and stiffness (Matsubara et al., 1998), but also by certain investigations showing the efficacy of anti-inflammatory agents (statins) in reducing mortality in HFpEF patients (Liu et al., 2014; Alehagen et al., 2015; Marume et al., 2019). Moreover, it must be noted that the aforementioned systemic proinflammatory state is, in fact, also involved in the pathogenesis of HFrEF, whereby in addition to cardiomyocyte death secondary to an inciting stimulus, elevated levels of IL6 and TNF- $\alpha$ also mediate a reduction in NO-cGMP-PKG signaling that contributes to myocardial dysfunction (Paulus and Tschöpe, 2013). However, despite the apparent importance of inflammation in the pathogenesis of HFpEF (and HFrEF), a significant proportion of conducted clinical trials have also demonstrated the ineffectiveness of anti-inflammatory statins as well as vasoprotective ACE inhibitors (Fu et al., 2012) and angiotensin II receptor antagonists (Yusuf et al., 2003) in HFpEF cohorts, thereby somewhat diminishing the credibility of the systemic proinflammatory hypothesis.

\section{MULTI-ORGAN DISEASE HYPOTHESIS}

An alternative theory is the belief that HFpEF, rather than being a single disease, instead results from the interaction of multiple underlying physiological ailments. These include not only a reduction in diastolic function and cardiac reserves but also impairments in the renal and pulmonary systems (Borlaug, 2014), all of which collectively show significant inter-individual variations (Roh et al., 2017). The severity of these conditions is age-dependent (Parikh et al., 2018), and frailty (Tse et al., 2018; Zhang et al., 2018), a syndrome resulting from an age-related reduction in physiological function, itself has been linked with adverse outcomes in HF (Figure 1C). All in all, this notion of a differential phenotypic expression resulting from a complex interplay of multiple comorbidities is well-accepted, and likely accounts for the failure of conventional pharmacological therapies used in HFrEF to yield the same beneficial outcomes in HFpEF. Nonetheless, recent efforts have been made to construct animal models that closely mimic HFpEF phenotype. Obesity as well as salt-driven hypertensive models have both been used to accurately study respiratory muscle weakness and associated exercise intolerance in HFpEF. Seiler et al. describes salt-loaded hypertensive HFpEF rats with diaphragmatic muscle alterations secondary to elevated plasma levels of inflammatory cytokines (TNF- $\alpha$, IL-1 $\beta$, etc.), consistent with the systemic proinflammatory hypothesis (Seiler et al., 2016). Moreover, models of renal insufficiency-driven hypertension have also shown to present with LV hypertrophy and poor $\mathrm{LV}$ relaxation, reflective of the diastolic dysfunction related to HFpEF (Munagala et al., 2005). However, as previously stated, HFpEF is characterized by multiple comorbidities that interact to produce to the final phenotype. This cannot be replicated by animal models, which normally elucidate the mechanistic role of only one particular comorbidity (e.g., arterial hypertension, obesity, renal insufficiency, etc.) in the development of HFpEF. Whilst this approach allows for an understanding of the relationship between each individual comorbidity and HFpEF (Valero-Muñoz et al., 2017), it will likely serve to benefit only a subset of patients for whom the investigated comorbidity is the predominant factor contributing to disease pathogenesis.

\section{DYSSYNCHRONY}

Both the systemic proinflammatory state and multi-organ disease hypotheses encompass the role of left ventricular diastolic dysfunction (LVDD) in HFpEF development. LVDD has long been considered the major causative factor of HFpEF; however, many previous trials aiming to reduce long-term mortality by enhancing diastolic function, namely by improving LV relaxation and/or halting the progression of LV hypertrophy through the antagonism of the renin-angiotensin-aldosterone system, have failed to report favorable outcomes (Cleland et al., 2006; Massie et al., 2008). Such findings have warranted and fueled the search for other contributing mechanisms central to the pathogenesis of $\mathrm{HFpEF}$ for prospective targeting in the clinical setting. One such alternative that is becoming increasingly investigated is LV dyssynchrony (Figure 1D), which stems from the regional variations in the rate of contraction and relaxation of fibers in the myocardium, in turn resulting in impaired cardiac performance (Lee et al., 2011). Although it is said to exist in $\sim 30-40 \%$ of HFrEF patients (Liu et al., 2018), LV dyssynchrony and its severity have also now been associated with the development of HFpEF (Lee et al., 2011; Santos et al., 2014). Lee et al. showcased not only the existence of systolic and diastolic dyssynchrony at rest in an HFpEF cohort relative to normal controls but also the subsequent aggravation of dyssynchrony when HFpEF patients were exposed to dobutamine-induced hemodynamic stress (Lee 


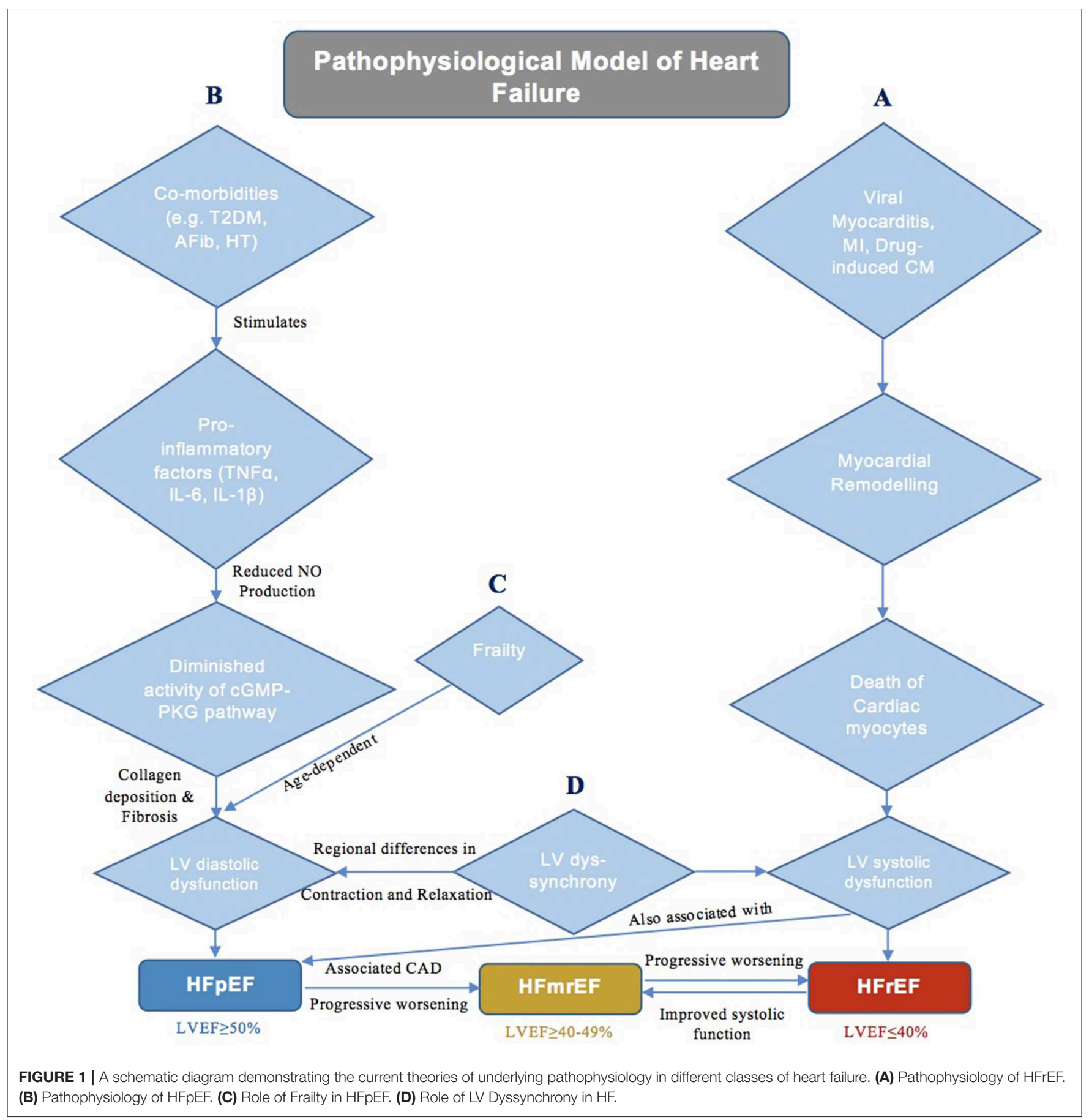

et al., 2010). Moreover, Morris et al. demonstrated that the development of LV dyssynchrony is, in fact, associated with subendocardial fibrosis that occurs secondary to a vast majority of comorbidities typically accompanying the HFpEF phenotype (Morris et al., 2012). Such dyssynchrony has been implicated in the development of systolic dysfunction in HFpEF patients, in which delayed myocardial activation diminishes pump efficiency (Cheng et al., 2009). In addition, it has also been postulated that asynchronous LV contraction leads to temporal variability in
LV relaxation, particularly during early diastole, wherein certain myocardial fibers relax later than others (Bonow et al., 1988). This subsequently decreases diastolic function, presenting as a reduction in passive LV filling and an increase in LV filling pressures (Morris et al., 2012). All in all, the dynamicity of mechanical dyssynchrony, along with the resulting systolic and diastolic dysfunction, are clearly important to the manifestation of HFpEF, and in turn provide reason as to why therapies that focus solely on the improvement of diastolic function 
without any regard for LV dyssynchrony have failed to yield fruitful outcomes.

\section{MECHANISMS OF HFmrEF}

In contrast to HFpEF and HFrEF, there is a relative paucity in literature discussing the phenotype of HFmrEF, and as such, this condition has often been referred to as the "middle child" in the HF family. The pathophysiology of HFmrEF, which accounts for $\sim 10-20 \%$ of all HF patients (Nadar and Tariq, 2018), is largely unknown. Current evidence indicates that HFmrEF may be the outcome of a progressive worsening in LV function in HFpEF patients, most notably observed in those with concomitant coronary artery disease, which is associated with a deterioration in LVEF (Lam and Solomon, 2014). An alternative possibility is that HFmrEF results from an improved systolic function in patients with HFrEF. This particular subset of HFmrEF is clinically relevant, as recovered systolic function in HF patients has been linked with reduced mortality and a more favorable long-term prognosis (Nadruz et al., 2016). Regardless of its pathway of development, many studies have presented HFmrEF as an intermediate phenotype with clinical characteristics and outcomes in between the other two classes of HF, albeit more closely related to HFrEF (He et al., 2009; Rickenbacher et al., 2017; Lund et al., 2018). Although such findings of an intermediate clinical profile would support the notion of HFmrEF as a distinct condition-a subset of neither HFpEF nor HFrEF-additional studies are still required for this to be confirmed and for the pathogenesis of HFmrEF to be further elucidated.

\section{CARDIAC IMAGING, BIOMARKERS, AND NETWORK ANALYSIS}

The heterogeneity of HF has necessitated the use of various techniques in cardiac imaging for diagnosis and prognosis assessment, namely transthoracic echocardiography (TTE), cardiac computerized tomography, and magnetic resonance imaging (Butler, 2007; Inamdar and Inamdar, 2016). Moreover, three-dimensional speckle tracking in echocardiography, a relatively recent development, has also proven to be an accurate, relatively efficient method for assessing LV function in research, thereby warranting its more frequent implementation in the clinical setting for the evaluation of HF (Luo et al., 2014; $\mathrm{Xu}$ et al., 2014). However, in addition to the aforementioned cardiac imaging methods, numerous biomarkers related to the pathological processes of HF, such as inflammation (Petersen and Felker, 2006; Bozkurt et al., 2010), myocardial mechanical stress, and fibrosis (Ahmad et al., 2014; López et al., 2015; Li et al., 2018), cardio-renal dysfunction (Senthong et al., 2017), and even the ovarian cancer marker cancer antigen-125 (Cheung et al., 2018), have been identified and investigated. Such biomarker studies perhaps provide some of the most important avenues through which the diversity of HF can be further understood. Recently, network analysis has been implemented as a tool to study different multi-marker interactions for the purpose of obtaining a more comprehensive overview of the distinct pathogenesis of the three classes of HF. Thus, far, this summative, allencompassing approach has somewhat unsurprisingly revealed multiple shared protein-protein based relationships among HFpEF, HFmrEF, and HFrEF. However, findings have also indicated varying biomarker signatures within the different HF groups. Most notably, HFpEF pathways were uniquely associated with markers of inflammation whilst HFrEF pathways were enriched with markers of cardiac stretch and cellular proliferation, both showing minimal overlapping with HFmrEF (Tromp et al., 2017, 2018). The identification of such specific biochemical interactions not only emphasizes the unique pathophysiology of the three types of HF but also provides insight into potential drug targets, and in turn, suggests the need for dynamic multi-marker screening for optimal risk stratification.

\section{PHENOMAPPING}

In addition to network analysis, another method that has more recently been implemented to assess the underlying heterogeneity in HF, amongst various other diseases, is "phenomapping," which involves machine-based learning to analyze large sums of phenotypic data in order to categorize patients into distinct subgroups based on a select number of clinical features (Katz et al., 2017). Katz et al. demonstrated the use of this technique to successfully identify distinct subgroups of hypertensive patients with a predisposition to HFpEF development owing to the presence of abnormal cardiac mechanical properties (Katz et al., 2017). Similarly, Shah et al. also used machine learning to stratify an HFpEF cohort according to a series of unrelated clinical phenotypic features found in the domains of patient demographics, physical characteristics, as well as laboratory, ECG and echocardiography parameters. This classification yielded three distinct "phenogroups" of HFpEF patients, all of whom presented with variable baseline data. Pheno-group \#1 was characterized by low levels of brain natriuretic peptide (BNP), which is typically associated with obesity. A lack of BNP promotes renal sodium and water retention, culminating in an increase in plasma volume, preload, and LV hypertrophy. As a result, such a "BNP deficiency syndrome" could likely serve as a possible explanation for the underlying etiology of diastolic dysfunction and subsequent HFpEF in this particular subset of patients. Pheno-group \#2 encompassed a phenotype of cardio-metabolic disease, which can disturb myocardial electrical and mechanical function, whilst pheno-group \#3 consisted of patients with the highest BNP levels, but concurrent right ventricular and renal dysfunction that predisposed to the highest risk of hospitalization and mortality (Shah et al., 2015; Shah, 2017). With the findings of these investigations, it is evident that phenomapping can serve as a tool to circumvent the problem of heterogeneity in HF by categorizing patients into distinct, clinically relevant clusters. The identification of such subgroups 
would subsequently allow for the development of patientspecific, targeted therapies that could potentially improve longterm prognosis.

\section{CONCLUSION}

The three classes of HF are all characterized by distinct pathophysiological processes, which in turn contribute to the heterogeneity in the expressed phenotype. Whilst there is currently less unknown about HFrEF, literature pertaining to HFpEF and the newly classified HFmrEF still incites many questions with respect to pathogenesis and optimal therapeutic strategies. Specifically, more prospective studies, as opposed to cross-sectional studies, that follow HFpEF and HFmrEF patients for a lengthy time period are required to assess whether or not the development of HF is truly, in fact, a continuum that progresses from HFpEF through to HFmrEF and eventually HFrEF. Overall, the diversity in the observed clinical features,

\section{REFERENCES}

Ahmad, T., Fiuzat, M., Neely, B., Neely, M. L., Pencina, M. J., Kraus, W. E., et al. (2014). Biomarkers of myocardial stress and fibrosis as predictors of mode of death in patients with chronic heart failure. JACC Heart Fail. 2, 260-268. doi: 10.1016/j.jchf.2013.12.004

Alehagen, U., Benson, L., Edner, M., Dahlström, U., and Lund, L. H. (2015). Association between use of statins and mortality in patients with heart failure and ejection fraction of $\geq 50$. Circ. Heart Fail. 8, 862-870. doi: 10.1161/CIRCHEARTFAILURE.115.002143

Bloom, M. W., Greenberg, B., Jaarsma, T., Januzzi, J. L., Lam, C. S. P., Maggioni, A. P., et al. (2017). Heart failure with reduced ejection fraction. Nat. Rev. Dis. Prim. 3:17058. doi: 10.1038/nrdp.2017.58

Bonow, R. O., Vitale, D. F., Bacharach, S. L., Maron, B. J., and Green, M. V. (1988). Effects of aging on asynchronous left ventricular regional function and global ventricular filling in normal human subjects. J. Am. Coll. Cardiol. 11, 50-58. doi: 10.1016/0735-1097(88)90166-0

Borlaug, B. A. (2014). The pathophysiology of heart failure with preserved ejection fraction. Nat. Rev. Cardiol. 11, 507-515. doi: 10.1038/nrcardio.2014.83

Bozkurt, B., Mann, D. L., and Deswal, A. (2010). Biomarkers of inflammation in heart failure. Heart Fail. Rev. 15, 331-341. doi: 10.1007/s10741-009-9140-3

Butler, J. (2007). The emerging role of multi-detector computed tomography in heart failure. J. Card. Fail. 13, 215-226. doi: 10.1016/j.cardfail.2006.11.014

Calderone, A., Thaik, C. M., Takahashi, N., Chang, D. L., and Colucci, W. S. (1998). Nitric oxide, atrial natriuretic peptide, and cyclic GMP inhibit the growthpromoting effects of norepinephrine in cardiac myocytes and fibroblasts. $J$. Clin. Invest. 101, 812-818. doi: 10.1172/JCI119883

Cheng, A., Helm, R. H., and Abraham, T. P. (2009). Pathophysiological mechanisms underlying ventricular dyssynchrony. Europace 11 (Suppl. 5), v10-14. doi: 10.1093/europace/eup272

Cheung, A., Gong, M., Bellanti, R., Ali-Hasan-Al-Saegh, S., Li, G., Roig, E., et al. (2018). Cancer antigen-125 and risk of atrial fibrillation: a systematic review and meta-analysis. Heart Asia 10:e10970. doi: 10.1136/heartasia-2017-01 0970

Cleland, J. G., Tendera, M., Adamus, J., Freemantle, N., Polonski, L., Taylor, J., et al. (2006). The perindopril in elderly people with chronic heart failure (PEP-CHF) study. Eur. Heart J. 27, 2338-2345. doi: 10.1093/eurheartj/ehl250

Elguindy, A., and Yacoub, M. H. (2012). Heart failure with preserved ejection fraction. Glob. Cardiol. Sci. Pract. 2012:10. doi: 10.5339/gcsp.2012.10

Fu, M., Zhou, J., Sun, A., Zhang, S., Zhang, C., Zou, Y., et al. (2012). Efficacy of ACE inhibitors in chronic heart failure with preserved ejection fractiona meta analysis of 7 prospective clinical studies. Int. J. Cardiol. 155, 33-38. doi: 10.1016/j.ijcard.2011.01.081 namely of HFpEF and HFmrEF, poses challenges to the creation of a universal treatment regimen that will fit all patients. As such, future management of these two conditions, in particular, will likely necessitate the implementation of a patient-specific method that targets unique metabolic derangements present in distinct patient subgroups. Although tools such as network analysis and machine-based learning that attempt to collate and find links between available data have proven to yield informative results, a greater understanding of the underlying mechanisms involved in the development of HFpEF and HFmrEF is still required before this individualistic approach can be employed holistically in the clinical setting.

\section{AUTHOR CONTRIBUTIONS}

All authors listed have made a substantial, direct and intellectual contribution to the work, and approved it for publication.
Gonzalez, A., Ravassa, S., Beaumont, J., Lopez, B., and Diez, J. (2011). New targets to treat the structural remodeling of the myocardium. J. Am. Coll. Cardiol. 58, 1833-1843. doi: 10.1016/j.jacc.2011.06.058

He, K. L., Burkhoff, D., Leng, W. X., Liang, Z. R., Fan, L., Wang, J., et al. (2009). Comparison of ventricular structure and function in Chinese patients with heart failure and ejection fractions $>55 \%$ versus $40 \%$ to $55 \%$ versus $<40 \%$. Am. J. Cardiol. 103, 845-851. doi: 10.1016/j.amjcard.2008.11.050

Inamdar, A. A., and Inamdar, A. C. (2016). Heart failure: diagnosis, management and utilization. J Clin Med 5:E62. doi: 10.3390/jcm5070062

Kao, D. P., Lewsey, J. D., Anand, I. S., Massie, B. M., Zile, M. R., Carson, P. E., et al. (2015). Characterization of subgroups of heart failure patients with preserved ejection fraction with possible implications for prognosis and treatment response. Eur. J. Heart Fail. 17, 925-935. doi: 10.1002/ejhf.327

Katz, D. H., Deo, R. C., Aguilar, F. G., Selvaraj, S., Martinez, E. E., Beussink-Nelson, L., et al. (2017). Phenomapping for the identification of hypertensive patients with the myocardial substrate for heart failure with preserved ejection fraction. J. Cardiovasc. Transl. Res. 10, 275-284. doi: 10.1007/s12265-017-9739-Z

Lam, C. S., and Solomon, S. D. (2014). The middle child in heart failure: heart failure with mid-range ejection fraction (40-50\%). Eur. J. Heart Fail. 16, 1049-1055. doi: 10.1002/ejhf.159

Lee, A. P., Song, J. K., Yip, G. W., Zhang, Q., Zhu, T. G., Li, C., et al. (2010). Importance of dynamic dyssynchrony in the occurrence of hypertensive heart failure with normal ejection fraction. Eur. Heart J. 31, 2642-2649. doi: 10.1093/eurheartj/ehq248

Lee, A. P., Zhang, Q., Yip, G., Fang, F., Liang, Y. J., Xie, J. M., et al. (2011). LV mechanical dyssynchrony in heart failure with preserved ejection fraction complicating acute coronary syndrome. JACC Cardiovasc. Imaging 4, 348-357. doi: 10.1016/j.jcmg.2011.01.011

Li, K. H. C., Gong, M., Li, G., Baranchuk, A., Liu, T., Wong, M. C. S., et al. (2018). Cancer antigen-125 and outcomes in acute heart failure: a systematic review and meta-analysis. Heart Asia 10:e011044. doi: 10.1136/heartasia-2018-011044

Liu, G., Zheng, X. X., Xu, Y. L., Ru, J., Hui, R. T., and Huang, X. H. (2014). Metaanalysis of the effect of statins on mortality in patients with preserved ejection fraction. Am. J. Cardiol. 113, 1198-1204. doi: 10.1016/j.amjcard.2013.12.023

Liu, S., Guan, Z., Jin, X., Meng, P., Wang, Y., Zheng, X., et al. (2018). Left ventricular diastolic and systolic dyssynchrony and dysfunction in heart failure with preserved ejection fraction and a narrow QRS complex. Int. J. Med. Sci. 15, 108-14. doi: 10.7150/ijms.21956

López, B., González, A., Ravassa, S., Beaumont, J., Moreno, M. U., San Jos,é, G., et al. (2015). Circulating biomarkers of myocardial fibrosis: the need for a reappraisal. J. Am. Coll. Cardiol. 65, 2449-2456. doi: 10.1016/j.jacc.2015.04.026

Lund, L. H., Claggett, B., Liu, J., Lam, C. S., Jhund, P. S., Rosano, G. M., et al. (2018). Heart failure with mid-range ejection fraction in CHARM: characteristics, 
outcomes and effect of candesartan across the entire ejection fraction spectrum. Eur. J. Heart Fail. 20, 1230-1239. doi: 10.1002/ejhf.1149

Luo, X. X., Fang, F., Lee, A. P., Sun, J. P., Li, S., Zhang, Z. H., et al. (2014). What can three-dimensional speckle-tracking echocardiography contribute to evaluate global left ventricular systolic performance in patients with heart failure? Int. J. Cardiol. 172, 132-137. doi: 10.1016/j.ijcard.2013.12.314

Marume, K., Takashio, S., Nagai, T., Tsujita, K., Saito, Y., Yoshikawa, T., et al. (2019). Effect of statins on mortality in heart failure with preserved ejection fraction without coronary artery disease - report from the JASPER study. Circ. J. 83, 357-367. doi: 10.1253/circj.CJ-18-0639

Massie, B. M., Carson, P. E., Mcmurray, J. J., Komajda, M., Mckelvie, R., Zile, M. R., et al. (2008). Irbesartan in patients with heart failure and preserved ejection fraction. N. Engl. J. Med. 359, 2456-2467. doi: 10.1056/NEJMoa0805450

Matsubara, B. B., Matsubara, L. S., Zornoff, L. A., Franco, M., and Janicki, J. S. (1998). Left ventricular adaptation to chronic pressure overload induced by inhibition of nitric oxide synthase in rats. Basic Res. Cardiol. 93, 173-181. doi: $10.1007 / \mathrm{s} 003950050084$

Morris, D. A., Vaz Pérez, A., Blaschke, F., Eichstädt, H., Ozcelik, C., and Haverkamp, W. (2012). Myocardial systolic and diastolic consequences of left ventricular mechanical dyssynchrony in heart failure with normal left ventricular ejection fraction. Eur. Heart J. Cardiovasc. Imaging 13, 556-567. doi: 10.1093/ehjci/jes042

Munagala, V. K., Hart, C. Y., Burnett, J. C., Meyer, D. M., and Redfield, M. M. (2005). Ventricular structure and function in aged dogs with renal hypertension: a model of experimental diastolic heart failure. Circulation 111, 1128-1135. doi: 10.1161/01.CIR.0000157183.21404.63

Nadar, S. K., and Tariq, O. (2018). What is heart failure with mid-range ejection fraction? A new subgroup of patients with heart failure. Card Fail Rev 4, 6-8. doi: 10.15420/cfr.2018:7:2

Nadruz, W., West, E., Santos, M., Skali, H., Groarke, J. D., Forman, D. E., et al. (2016). Heart failure and midrange ejection fraction: implications of recovered ejection fraction for exercise tolerance and outcomes. Circ. Heart Fail. 9:e002826. doi: 10.1161/CIRCHEARTFAILURE.115.002826

Parikh, K. S., Sharma, K., Fiuzat, M., Surks, H. K., George, J. T., Honarpour, N., et al. (2018). Heart failure with preserved ejection fraction expert panel report: current controversies and implications for clinical trials. JACC Heart Fail. 6, 619-632. doi: 10.1016/j.jchf.2018.06.008

Paulus, W. J., and Tschöpe, C. (2013). A novel paradigm for heart failure with preserved ejection fraction: comorbidities drive myocardial dysfunction and remodeling through coronary microvascular endothelial inflammation. J. Am. Coll. Cardiol. 62, 263-271. doi: 10.1016/j.jacc.2013.02.092

Petersen, J. W., and Felker, G. M. (2006). Inflammatory biomarkers in heart failure. Congest. Heart Fail. 12, 324-328. doi: 10.1111/j.1527-5299.2006.05595.x

Ponikowski, P., Voors, A. A., Anker, S. D., Bueno, H., Cleland, J. G., Coats, A. J., et al. (2016). 2016 ESC Guidelines for the diagnosis and treatment of acute and chronic heart failure: the task force for the diagnosis and treatment of acute and chronic heart failure of the European Society of Cardiology (ESC). Developed with the special contribution of the Heart Failure Association (HFA) of the ESC. Eur. J. Heart Fail. 18, 891-975. doi: 10.1002/ejhf.592

Rickenbacher, P., Kaufmann, B. A., Maeder, M. T., Bernheim, A., Goetschalckx, K., Pfister, O., et al. (2017). Heart failure with mid-range ejection fraction: a distinct clinical entity? Insights from the Trial of Intensified versus standard Medical therapy in Elderly patients with Congestive Heart Failure (TIME-CHF). Eur. J. Heart Fail. 19, 1586-1596. doi: 10.1002/ejhf.798

Roh, J., Houstis, N., and Rosenzweig, A. (2017). Why don't we have proven treatments for HFpEF? Circ. Res. 120, 1243-1245. doi: 10.1161/CIRCRESAHA.116.310119

Santos, A. B., Kraigher-Krainer, E., Bello, N., Claggett, B., Zile, M. R., Pieske, B., et al. (2014). Left ventricular dyssynchrony in patients with heart failure and preserved ejection fraction. Eur. Heart J. 35, 42-47. doi: 10.1093/eurheartj/eht427
Savarese, G., and Lund, L. H. (2017). Global public health burden of heart failure. Card Fail. Rev. 3, 7-11. doi: 10.15420/cfr.2016:25:2

Seiler, M., Bowen, T. S., Rolim, N., Dieterlen, M. T., Werner, S., Hoshi, T., et al. (2016). Skeletal muscle alterations are exacerbated in heart failure with reduced compared with preserved ejection fraction: mediated by circulating cytokines? Circ Heart Fail 9:e003027. doi: 10.1161/CIRCHEARTFAILURE.116.0 03027

Senthong, V., Kirsop, J. L., and Tang, W. H. (2017). Clinical phenotyping of heart failure with biomarkers: current and future perspectives. Curr. Heart Fail. Rep. 14, 106-116. doi: 10.1007/s11897-017-0321-4

Shah, S. J. (2017). Precision medicine for heart failure with preserved ejection fraction: an overview. J. Cardiovasc. Transl. Res. 10, 233-244. doi: 10.1007/s12265-017-9756-y

Shah, S. J., Katz, D. H., Selvaraj, S., Burke, M. A., Yancy, C. W., Gheorghiade, M., et al. (2015). Phenomapping for novel classification of heart failure with preserved ejection fraction. Circulation 131, 269-279. doi: 10.1161/CIRCULATIONAHA.114.010637

Tromp, J., Khan, M. A., Klip, I. T., Meyer, S., De Boer, R. A., Jaarsma, T., et al. (2017). Biomarker profiles in heart failure patients with preserved and reduced ejection fraction. J. Am. Heart. Assoc. 6:e003989. doi: 10.1161/JAHA.116.003989

Tromp, J., Westenbrink, B. D., Ouwerkerk, W., Van Veldhuisen, D. J., Samani, N. J., Ponikowski, P., et al. (2018). Identifying pathophysiological mechanisms in heart failure with reduced versus preserved ejection fraction. J. Am. Coll. Cardiol. 72, 1081-1090. doi: 10.1016/j.jacc.2018. 06.050

Tse, G., Gong, M., Wong, S. H., Wu, W. K. K., Bazoukis, G., Lampropoulos, K., et al. (2018). Frailty and clinical outcomes in advanced heart failure patients undergoing left ventricular assist device implantation: a systematic review and meta-analysis. J. Am. Med. Dir. Assoc. 19, 255-261.e251. doi: 10.1016/j.jamda.2017.09.022

Valero-Muñoz, M., Backman, W., and Sam, F. (2017). Murine models of heart failure with preserved ejection fraction: a "fishing expedition". JACC Basic Transl. Sci. 2, 770-789. doi: 10.1016/j.jacbts.2017.07.013

Van Linthout, S., and Tschöpe, C. (2017). Inflammation - cause or consequence of heart failure or both? Curr. Heart Fail. Rep. 14, 251-265. doi: 10.1007/s11897-017-0337-9

Xu, T. Y., Sun, J. P., Lee, A. P., Yang, X. S., Qiao, Z., Luo, X., et al. (2014). Threedimensional speckle strain echocardiography is more accurate and efficient than 2D strain in the evaluation of left ventricular function. Int. J. Cardiol. 176, 360-366. doi: 10.1016/j.ijcard.2014.07.015

Yusuf, S., Pfeffer, M. A., Swedberg, K., Granger, C. B., Held, P., Mcmurray, J. J., et al. (2003). Effects of candesartan in patients with chronic heart failure and preserved left-ventricular ejection fraction: the CHARM-Preserved Trial. Lancet 362, 777-781. doi: 10.1016/S0140-6736(03)14285-7

Zhang, Y., Yuan, M., Gong, M., Tse, G., Li, G., and Liu, T. (2018). Frailty and clinical outcomes in heart failure: a systematic review and meta-analysis. J. Am. Med. Dir. Assoc. 19, 1003-1008.e1001. doi: 10.1016/j.jamda.2018.0 6.009

Conflict of Interest Statement: The authors declare that the research was conducted in the absence of any commercial or financial relationships that could be construed as a potential conflict of interest.

Copyright (c) 2019 Lakhani, Leung, Tse and Lee. This is an open-access article distributed under the terms of the Creative Commons Attribution License (CC BY). The use, distribution or reproduction in other forums is permitted, provided the original author(s) and the copyright owner(s) are credited and that the original publication in this journal is cited, in accordance with accepted academic practice. No use, distribution or reproduction is permitted which does not comply with these terms. 